\title{
Fixed-Time Sliding-Mode Fault-Tolerant Control of Waste Heat Power Generator Systems
}

\author{
Zhi Wang, ${ }^{1,2}$ Yateng Bai, ${ }^{1,2}$ Jin Xie,, ${ }^{1,2}$ Zhijie Li $\mathbb{D}^{1,2}$ Caoyuan Ma $\mathbb{D}^{1,2}$ Pengjuan Liu, ${ }^{1,2}$ \\ and Yong Zhang ${ }^{1,2}$ \\ ${ }^{1}$ Jiangsu Province Laboratory of Electrical and Automation Engineering for Coal Mining, China University of Mining and \\ Technology, Xuzhou, Jiangsu 221116, China \\ ${ }^{2}$ China University of Mining and Technology, Xuzhou, Jiangsu 221116, China
}

Correspondence should be addressed to Caoyuan Ma; mcaoyuan@cumt.edu.cn

Received 3 May 2018; Accepted 31 May 2018; Published 15 July 2018

Academic Editor: Diyi Chen

Copyright (c) 2018 Zhi Wang et al. This is an open access article distributed under the Creative Commons Attribution License, which permits unrestricted use, distribution, and reproduction in any medium, provided the original work is properly cited.

\begin{abstract}
In order to overcome disturbances such as the instability of internal parameters or the actuator fault, the time-varying proportionalintegral sliding-mode surface is defined for coordinated control of the excitation generator and the steam valve of waste heat power generation units, and a controller based on sliding-mode function is designed which makes the system stable for a limited time and gives it good performance. Based on this, a corresponding fault estimation law is designed for specific faults of systems, and a sliding-mode fault-tolerant controller is constructed based on the fixed-time control theory so that the systems can still operate stably when an actuator fault occurs and have acceptable performance. The simulation results show that the tracking error asymptotically tends to be zero, and the fixed-time sliding-mode fault-tolerant controller can obviously improve the dynamic performance of the system.
\end{abstract}

\section{Introduction}

Observations on the long-term operation of waste heat power generation systems have shown that coordinated control of the excitation generator and the steam valve can significantly improve the limit of the transient stability of the system [1, 2], effectively improving the dynamic response following system failure, and reducing lowfrequency oscillations. Therefore, generator control technology must be developed according to the coordinated control of the steam valve and excitation, and this project requires a combination of valve control and generator excitation control for its implementation.

However, it is very difficult to achieve precise control for synchronous generators with strong nonlinearity.
Many kinds of control schemes have been put forward on the basis of a large number of domestic and foreign research. The control system of a turbogenerator set has been developed to a certain extent, but there is no perfect control means, and there are always shortcomings to some extent.

In research on the theory of intelligent control, a series of new techniques of coordinated control have been proposed. After years of theoretical and technical advancements, they have achieved good results in the context of the coordinated control of the excitation generator and steam valve. Neural networks are very appropriate for coordinated control because of its self-learning and selforganization. In [3], neural networks are used to design the controller, which can quickly solve the coordinated 
control problem. In [4], the neural network inverse system has been applied to the system by analyzing its reversibility. Fuzzy control has also been widely applied to coordinated control [5-7]. But on the whole, using intelligent methods in coordinated control is not so mature, which needs more study.

Sliding-mode variable-structure control has the advantages of fast response, simple design process, good robustness, and some advantages in dealing with nonlinear problems [8]. It has been applied to a nonholonomic mobile manipulator system [9], where the system had uncertainties in the kinematic mobile manipulator model and drive motor parameters, external disturbances, and nonmodeled dynamics, which is typical for many nonlinear uncertain systems. It is robust against the internal and external parameters of disturbance systems [10].

However, at present, the sliding-mode variablestructure control is mostly finite-time sliding-mode variable-structure control, which is limited to the limitation of finite-time stability. The stability of the system is affected by the initial conditions of the system, and it is generally difficult to obtain accurate parameters in the power system at the initial conditions, with the fixedtime stability being a generalization of the finite-time stability. It has all the advantages of finite-time control, and fixed-time control can not only guarantee the upper bound of the stable time range, but also have stronger robustness and anti-interference ability. The most important thing is the global uniform asymptotic stability of the system under any initial condition.

Technical experience has indicated, however, that a conventional feedback control design for complex systems such as waste heat power generator systems results in unsatisfactory performance, or even instability, in the event of malfunctions in actuators [11]. To circumvent such weaknesses, new approaches to control system design have emerged with the purpose of tolerating component malfunctions while maintaining desirable stability and performance properties. These types of control systems are often known as fault-tolerant control systems (FTCSs). FTCSs are control systems that possess the ability to accommodate component faults automatically. The main task to be tackled in achieving fault tolerance is the design of a controller with a suitable structure to guarantee stability and satisfactory performance, not only when all components are operational but also in the event that actuators malfunction [11-14]. Ideally, in the presence of faults, the closed-loop system should be capable of maintaining its safe and reliable operation while preserving system stability [15].

On the basis of summarizing the current research status of residual heat generator control, this paper makes a comprehensive and in-depth study on the excitation and valve coordination control system of a waste heat generating set, establishes a mathematical model of the residual heat generator set, and uses a fixed-time faulttolerant control based on a sliding-mode variable structure for the existing actuator failures. The controller controls the excitation and valve-coordinated control system, thus restraining the disturbance and enabling the system to run stably on the premise of acceptable performance.

\section{Excitation and Steam Valve Coordination Control System Model for Thermal Generator Units}

Consider the mathematical model of an excitation and steam valve coordination control system model as follows:

$$
\begin{aligned}
\dot{\delta} & =(\omega-1) \omega_{0}, \\
\dot{\omega} & =\frac{\omega_{0}}{H} P_{H}+\frac{\omega_{0}}{H} \cdot C_{M L} P_{m 0}-\frac{\omega_{0}}{H} \frac{U_{s} E_{q}^{\prime}}{x_{d} \prime} \sin \delta-\frac{D}{H}(\omega-1) \omega_{0}, \\
\dot{E}_{q}^{\prime} & =-\frac{1}{T_{d 0}^{\prime}}\left[E_{q}^{\prime}-\left(\frac{\left(x_{d}-x_{d}^{\prime}\right) U_{s}}{x_{d}{ }^{\prime}} \cos \delta-u\right)\right], \\
\dot{P}_{H} & =-\frac{P_{H}}{T_{H} \sum}+\frac{C_{H}}{T_{H} \sum} P_{m 0}+\frac{C_{H}}{T_{H} \sum} u^{\prime},
\end{aligned}
$$

where $\delta$ is the power angle, $\omega$ is the rotating speed, $E_{q}^{\prime}$ is the transient emf, $P_{H}$ is the high-pressure mechanical power of the cylinder, $\omega_{0}$ is the generator synchronous angular speed, $C_{M L}$ is the medium-pressure and low-pressure mechanical power of the cylinder, $P_{m 0}$ is the initial input value of the mechanical power of the generator, $U_{s}$ is the bus voltage of the infinite capacity system, $D$ is the damping power coefficient, $H$ is the inertia constant of the generator rotor, $x_{d \Sigma}{ }^{\prime}$ is the transient integrated reactance, $T_{d 0}^{\prime}$ is the inertia time constant of the excitation winding, and $T_{H \Sigma}$ is the time constant of the high-pressure cylinder and the highpressure oil motive.

Let $P_{1}=P_{H}+C_{M L} P_{m 0}$. The system becomes

$$
\begin{aligned}
\dot{\delta} & =(\omega-1) \omega_{0}, \\
\dot{\omega} & =\frac{\omega_{0}}{H}\left(P_{1}-\frac{U_{s} E_{q}^{\prime}}{x_{d \Sigma}^{\prime}} \sin \delta-D(\omega-1)\right), \\
\dot{E}_{q}^{\prime} & =-\frac{1}{T_{d 0}^{\prime}}\left(E_{q}^{\prime}-\frac{\left(x_{d}-x_{d}^{\prime}\right) U_{s}}{x_{d \Sigma}^{\prime}} \cos \delta-u\right), \\
\dot{P}_{1} & =-\frac{P_{1}-C_{M L} P_{m 0}}{T_{H} \sum}+\frac{C_{H}}{T_{H} \sum} P_{m 0}+u^{\prime} .
\end{aligned}
$$

Parameter $P_{1}$ is controlled by the valve control parameter, $u^{\prime}$, and is unrelated to other state quantities and control quantities. It is related only to the system parameters, which are set as control quantity 1 , and the output voltage, $u$, of the excitation winding, which is set as control quantity 2 . At the same time, the actuator failures are considered. Then, the system becomes 


$$
\begin{aligned}
\dot{\delta} & =(\omega-1) \omega_{0}, \\
\dot{\omega} & =\frac{\omega_{0}}{H}\left(\left(P_{1}+d_{1}\right)-\frac{U_{s} E_{q}^{\prime}}{x_{d \Sigma}^{\prime}} \sin \delta-D(\omega-1)\right), \\
\dot{E}_{q}^{\prime} & =-\frac{1}{T_{d 0}^{\prime}}\left(E_{q}^{\prime}-\frac{\left(x_{d}-x_{d}^{\prime}\right) U_{s}}{x_{d \Sigma}^{\prime}} \cos \delta-\left(u+d_{2}\right)\right),
\end{aligned}
$$

where $d_{1}$ is the fault of the steam valve and $d_{2}$ is the fault of the excitation generator.

Let $D_{1}=\omega_{0} d_{1} / H, D_{2}=d_{2} / T_{d 0}^{\prime}$ and $\left\|\dot{D_{1}}\right\|<l_{1},\left\|\dot{D_{2}}\right\|<l_{2}$. $l_{1}, l_{2}$ is a constant, and $l_{1}>0, l_{2}>0$, thus, the system becomes

$$
\begin{aligned}
\dot{\delta} & =(\omega-1) \omega_{0}, \\
\dot{\omega} & =\frac{\omega_{0}}{H}\left(P_{1}-\frac{U_{s} E_{q}^{\prime}}{x_{d \Sigma}^{\prime}} \sin \delta-D(\omega-1)\right)+D_{1}, \\
\dot{E}_{q}^{\prime} & =-\frac{1}{T_{d 0}^{\prime}}\left(E_{q}^{\prime}-\frac{\left(x_{d}-x_{d}^{\prime}\right) U_{s}}{x_{d \Sigma}^{\prime}} \cos \delta-u\right)+D_{2} .
\end{aligned}
$$

Remark 1. $D$ is bounded and $\dot{D}$ is also bounded; adding $D$ does not affect the existence of the solution of the original differential equation. When the actuator fault function $D$ is a constant value, $\dot{D}_{1}=0$ and $\dot{D}_{2}=0$. When the actuator fault function $D$ is time-varying, considering $\left\|\dot{D}_{1}\right\|<d_{1},\left\|\dot{D}_{2}\right\|<$ $d_{2}, d_{1}>0, d_{2}>0$ are normal scalars.

If $f_{1}, f_{2}$ satisfied the function, then

(1) $f_{1}, f_{2}$ are continuous on $R$;

(2) $f_{1}, f_{2}$ are on $R$ to satisfy the Lipschitz condition.

That is, the continuity of functions is to guarantee the existence of equation solutions, while the Lipschitz condition is to ensure the uniqueness of solutions.

\section{Controller Designed for the Excitation and Steam Valve Coordination Control System Model}

For the design of the controller, we provide the definition of fixed-time stability and related lemmas.

Definition 1 (see [16]). Consider the following dynamic system:

$$
\dot{x}=f(x),
$$

where $x \in R^{n}$ is the system's state and $f$ is a smooth nonlinear function. If there is a fixed settling time, $T_{0}$, under any initial condition, the magnitude of $T_{0}$ is independent of the initial conditions.

For example, $\lim _{t \rightarrow T_{0}}\|x(t)\|=0$ and $x(t) \equiv 0$. If $t \geq T_{0}$, the system in (5) is said to be fixed-time stable.
Lemma 1 (see [17]). If there is a continuous function $V(t)$ : $[0, \infty) \rightarrow[0, \infty)$, wherein

(1) $V$ is positive definite,

(2) there are real numbers $c>0$ and $0<\rho<1$, satisfying

$$
\dot{V}(t) \leq-c V^{\rho}(t), \quad t \geq t_{0},
$$

then, when $t \geq t^{*}, V(t)=0$, the system is stable in

$$
t^{*}=t_{0}+\frac{V^{1-p}\left(t_{0}\right)}{c(1-\rho)} .
$$

Lemma 2 (see [18]). If there is a continuous unbounded function $V: R^{n} \rightarrow R_{+} \cup|0|$, where

(1) $V(x)=0 \Leftrightarrow x=0$,

(2) For $\alpha, \beta, p=1-1 / 2 \gamma, q=1+1 / 2 \gamma, \gamma>1$, when $x(t)$ satisfies the following inequality

$$
D * V(x(t)) \leq-\alpha V^{p}(x(t))-\beta V^{q}(x(t)),
$$

where $D * V(x(t))$ represents the upper-right derivative of function $V(x(t))$, the system is said to be stable, and the time required for it to stabilize is given by

$$
T\left(x_{0}\right) \leq T_{\max }:=\frac{\pi \gamma}{\sqrt{\alpha \beta}}, \quad \forall x_{0} \in R^{N} .
$$

Lemma 3 (see [7]). If nonzero positive real numbers $x_{2}, x_{1}$, $\ldots, x_{n} \geq 0$, then,

$$
\begin{aligned}
& \sum_{i=1}^{N} x_{i}^{\eta} \geq\left(\sum_{i=1}^{N} x_{i}\right)^{\eta}, \quad 0<\eta \leq 1, \\
& \sum_{i=1}^{N} x_{i}^{\theta} \geq N^{1-\theta}\left(\sum_{i=1}^{N} x_{i}\right)^{\theta}, \quad \theta>1 .
\end{aligned}
$$

This is the process for designing the controller. The switching function is first designed. We set the system output tracking target $\delta_{d}, \omega_{d}, E_{q d}^{\prime}$, and define the controlled magnitude of error as follows:

$$
\begin{aligned}
e_{\delta} & =\delta-\delta_{d}, \\
e_{\omega} & =\omega-\omega_{d}, \\
e_{E_{q}^{\prime}} & =E_{q}^{\prime}-E_{q d}^{\prime} .
\end{aligned}
$$

To extend robustness to the entire system and eliminate the steady state error, an integral term is added to the linear sliding surface to construct the following integral sliding surface:

$$
\begin{aligned}
& s_{1}=0.5\left(e_{\delta}+\int_{0}^{t} e_{\delta}(\tau) d \tau\right)+\left(e_{\omega}+\int_{0}^{t} e_{\omega}(\tau) d \tau\right), \\
& s_{2}=0.5\left(e_{\delta}+\int_{0}^{t} e_{\delta}(\tau) d \tau\right)+\left(e_{E_{q}^{\prime}}+\int_{0}^{t} e_{E_{q}^{\prime}}(\tau) d \tau\right), \\
& s=s_{1}+s_{2} .
\end{aligned}
$$


Once the design of the sliding surface is complete, we need to construct a suitable control law, and design the following:

$$
\begin{aligned}
& P_{1}=-\left(0.5 \cdot\left((\omega-1) \omega_{0}+\delta-\delta_{d}\right)+\frac{\omega_{0}}{H} \cdot\left(-\frac{U_{s} E_{q}^{\prime}}{x_{d \Sigma}^{\prime}} \sin \delta-D(\omega-1)+\omega-\omega_{d}\right)+k_{1} \cdot s_{1}+\widehat{D}_{1}+k \operatorname{sign}(s)|s|^{\alpha}+k s i g n(s)|s|^{\beta}\right) \cdot \frac{H}{\omega_{0}}, \\
& u=-\left(0.5 \cdot\left((\omega-1) \omega_{0}+\delta-\delta_{d}\right)-\frac{1}{T_{d 0}^{\prime}}\left(E_{q}^{\prime}-\frac{\left(x_{d}-x_{d}^{\prime}\right) U_{s}}{x_{d \Sigma}^{\prime}} \cos \delta\right)+E_{q}^{\prime}-E_{q d}^{\prime}+k_{1} \cdot s_{2}+\widehat{D}_{2}+k \operatorname{sign}(s)|s|^{\alpha}+k \operatorname{sign}(s)|s|^{\beta}\right) \cdot T_{d 0^{\prime}}^{\prime}
\end{aligned}
$$

where $\delta_{d}, \omega_{d}$, and, $E_{q d}^{\prime}$ are the rated value, $0<\alpha<1, \beta>1$, the feedback gain of the tuning parameter of the terminal attractor $k>0$, and

$$
\dot{k}=2\left[|s|^{\alpha+1}+|s|^{\beta+1}\right]-(k-g)^{\alpha}-(k-g)^{\beta},
$$

where $g$ is any normal number.

Theorem 1. Considering the waste heat power generator system (3), using the designed switching function (12) and control law (13), and applying the fixed-time stability theory, the system will reach the stable state in a fixed time and become steady.

Proof 1. The Lyapunov function is selected as follows:

$$
\begin{aligned}
V= & \frac{1}{2} s^{2}+\frac{1}{2}(k-g)^{2}, \\
\dot{V}= & s \cdot \dot{s}+(k-g) \dot{k} \\
= & s \cdot\left(\dot{\delta}+\delta-\delta_{d}+\dot{\omega}+\omega-\omega_{d}+\dot{E}_{q}^{\prime}+E_{q}^{\prime}-E_{q d}^{\prime}\right)+(k-g) \dot{k} \\
= & s \cdot\left\{(\omega-1) \omega_{0}+\delta-\delta_{d}+\left(\omega_{0} / H\right)\right. \\
& \cdot\left[P_{1}-\left(U_{s} / x_{d} \sum E_{q} \sin \sigma-D(\omega-1)\right]+\omega-\omega_{d}\right. \\
& \quad\left(1 / T_{d 0}^{\prime}\right)\left(E_{q}^{\prime}-\left(\left(x_{d}-x_{d}^{\prime}\right) U_{s} / x_{d} \sum^{\prime}\right) \cos \delta-u\right) \\
& \left.+E_{q}-E_{q d}\right\}+(k-g) \dot{k} \\
= & \cdot\left\{(\omega-1) \omega_{0}+\delta-\delta_{d}-1 / 2\left[(\omega-1) \omega_{0}+\delta-\delta_{d}\right]-k_{1} s_{1}\right. \\
& \quad-k s i g n(s)|s|^{\alpha}-k s i g n(s)|s|^{\beta}-1 / 2\left[(\omega-1) \omega_{0}+\delta-\delta_{d}\right] \\
& \left.\quad k_{1} s_{2}-k s i g n(s)|s|^{\alpha}-k s i g n(s)|s|^{\beta}\right\}+(k-g) \dot{k} \\
= & 2 \cdot s\left[-1 / 2 k_{1} s-k s i g n(s)|s|^{\alpha}-k s i g n(s)|s|^{\beta}\right]+(k-g) \dot{k} \\
= & -k_{1} s^{2}-2 k|s|^{\alpha+1}-2 k|s|^{\beta+1}+2(k-g)\left(|s|^{\alpha+1}+|s|^{\beta+1}\right) \\
& -(k-g)^{\alpha+1}-(k-g)^{\beta+1} ;\left(k_{1}>0\right)<-2 g|s|^{\alpha+1} \\
& -2 g|s|^{\beta+1}-(k-g)^{\alpha+1}-(k-g)^{\beta+1} \\
= & -2^{1 / 2(\alpha+1)} \cdot 2 g \cdot\left(1 / 2 s^{2}\right)^{1 / 2(\alpha+1)}-2^{1 / 2(\alpha+1)} \\
& \cdot\left[1 / 2(k-g)^{2}\right]^{1 / 2(\alpha+1)}-2^{1 / 2(\beta+1)} \cdot 2 g \cdot\left(1 / 2 s^{2}\right)^{1 / 2(\beta+1)} \\
& -2^{1 / 2(\beta+1)} \cdot\left[1 / 2(k-g)^{2}\right]^{1 / 2(\beta+1)}
\end{aligned}
$$

$$
\begin{aligned}
< & -m\left\{\left(1 / 2 s^{2}\right)^{1 / 2(\alpha+1)}+\left[1 / 2(k-g)^{2}\right]^{1 / 2(\alpha+1)}\right\} \\
& -n\left\{\left(1 / 2 s^{2}\right)^{1 / 2(\beta+1)}+\left[1 / 2(k-g)^{2}\right]^{1 / 2(\beta+1)}\right\} \\
m= & \min \left\{2 \cdot 2^{1 / 2(\alpha+1)} g, 2^{1 / 2(\alpha+1)}\right\}, \\
n= & \min \left\{2 \cdot 2^{1 / 2(\beta+1)} g, 2^{1 / 2(\beta+1)}\right\} .
\end{aligned}
$$

Thus, according to Lemma 3,

$$
\begin{aligned}
\dot{V} \leq & -m\left\{\left(\frac{1}{2} s^{2}\right)+\left[\frac{1}{2}(k-g)^{2}\right]\right\}^{(1 / 2)(\alpha+1)} \\
& -2^{1-(\beta / 2)} n\left\{\left(\frac{1}{2} s^{2}\right)+\left[\frac{1}{2}(k-g)^{2}\right]\right\}^{(1 / 2)(\beta+1)} \\
= & -m V^{(1 / 2)(\alpha+1)}-2^{1-(\beta / 2)} n V^{(1 / 2)(\beta+1)} .
\end{aligned}
$$

Therefore, it follows from Lemma 1 , where $0<\alpha<1$, $\beta>1$, and $k$ is any constant greater than zero. The proof is complete.

From Lemma 2, we can get a stable fixed time:

$$
\begin{aligned}
T & \leq \frac{1}{m(1-(\alpha+1) / 2)}+\frac{1}{2^{1-\beta / 2} n((\beta+1) / 2-1)} \\
& =\frac{2}{m(1-\alpha)}+\frac{2^{(\beta+1) / 2}}{n(\beta-1)} .
\end{aligned}
$$

For the designed control law, the following theorem is given.

Theorem 2. Considering the waste heat power generator system (3), using the designed switching function (12) and control law (13), and applying the change of the sliding surface to estimate the fault, the system will reach the stable state in a limited time and become steady.

Proof 2. Let one select the Lyapunov function as follows: 
TABLE 1: System parameters.

\begin{tabular}{lccc}
\hline Parameter & Value & Parameter & Value \\
\hline$\omega_{0}$ & 1 & $T_{d 0}^{\prime}$ & 1.3201 \\
$U_{s}$ & 0.995 & $x_{d}^{\prime}$ & 1.165 \\
$H$ & 0.3407 & $x_{d}$ & 1.8 \\
$D$ & 0.1 & $x_{d}^{\prime}$ & 0.3 \\
\hline
\end{tabular}

$$
V=0.5 s^{2}+0.5 \tilde{D}_{1} \Gamma_{1}^{-1} \tilde{D}_{1}+0.5 \tilde{D}_{2} \Gamma_{2}^{-1} \tilde{D}_{2}+0.5(k-g)^{2},
$$

where,

$$
\begin{aligned}
& \tilde{D}_{1}=D_{1}-\widehat{D}_{1}, \\
& \tilde{D}_{2}=D_{2}-\widehat{D}_{2} .
\end{aligned}
$$

Definition:

$$
\begin{aligned}
& \dot{\hat{D}}_{1}=\Gamma_{1} s, \\
& \dot{\hat{D}}_{2}=\Gamma_{2} s,
\end{aligned}
$$

then,

$$
\begin{aligned}
\dot{V}=s \cdot\{ & (\omega-1) \omega_{0}+\delta-\delta_{d}+\frac{\omega_{0}}{H}\left[P_{1}-\frac{U_{s}}{x_{d} \Sigma} E_{q} \sin \sigma-D(\omega-1)\right] \\
& \left.+\omega-\omega_{d}-\frac{1}{T_{d 0}^{\prime}}\left(E_{q}^{\prime}-\frac{\left(x_{d}-x_{d}^{\prime}\right) U_{s}}{x_{d}^{\prime} \Sigma} \cos \delta-u\right)+E_{q}-E_{q d}\right\} \\
& +(k-g) \dot{k} \\
=s \cdot & (\omega-1) \omega_{0}+\delta-\delta_{d}-\frac{1}{2}\left[(\omega-1) \omega_{0}+\delta-\delta_{d}\right]+D_{1}-\widehat{D}_{1} \\
& \quad k_{1} s_{1}-k s i g n(s)|s|^{\alpha}-k s i g n(s)|s|^{\beta}-\frac{1}{2}\left[(\omega-1) \omega_{0}+\delta-\delta_{d}\right] \\
& \left.+D_{2}-\widehat{D}_{2}-k_{1} s_{2}-k \operatorname{sign}(s)|s|^{\alpha}-k s i g n(s)|s|^{\beta}\right\}+(k-g) \dot{k} \\
= & -k_{1} s^{2}+\tilde{D}_{1} s+\tilde{D}_{1} \Gamma_{1}^{-1} \dot{\hat{D}}_{1}+\tilde{D}_{2} s+\tilde{D}_{2} \Gamma_{2}^{-1} \dot{\hat{D}}_{2}-2 k|s|^{\alpha+1} \\
& -2 k|s|^{\beta+1}+(k-g) \dot{k} \\
= & -k_{1} s^{2}+\tilde{D}_{1} \Gamma_{1}^{-1} \hat{\tilde{D}}_{1}+\tilde{D}_{2} \Gamma_{2}^{-1} \dot{\hat{D}}_{2}-2 k|s|^{\alpha+1}-2 k|s|^{\beta+1}(k-g) \dot{k},
\end{aligned}
$$

then,

$$
\begin{gathered}
-k_{1} s^{2}+s \tilde{D}_{1}+\tilde{D}_{1} \Gamma_{1}^{-1} \dot{\tilde{D}}_{1}+s \tilde{D}_{2}+\tilde{D}_{2} \Gamma_{2}^{-1} \dot{\tilde{D}}_{2} \\
=-k_{1} s^{2}+\tilde{D}_{1} \Gamma_{1}^{-1} \dot{D}_{1}+\tilde{D}_{2} \Gamma_{2}^{-1} \dot{D}_{2} .
\end{gathered}
$$

When $k_{1}>0,-k_{1} s^{2}<0$, choosing $\Gamma_{1}$ and $\Gamma_{2}$ that are large enough to make $\tilde{D}_{1} \Gamma_{1}^{-1} \dot{D}_{1}+\tilde{D}_{2} \Gamma_{2}^{-1} \dot{D}_{2}$ small enough in this case will be $\tilde{D}_{1} \Gamma_{1}^{-1} \dot{D}_{1}+\tilde{D}_{2} \Gamma_{2}^{-1} \dot{D}_{2}<0$, then,
TABLE 2: Control parameters.

\begin{tabular}{lc}
\hline Parameter & Value \\
\hline$k_{1}$ & 8 \\
$\Gamma_{1}, \Gamma_{2}$ & 50 \\
$\delta_{d}, \omega_{d}, E_{q d}^{\prime}$ & 1 \\
\hline
\end{tabular}

$$
\begin{aligned}
\dot{V}< & -2 k|s|^{\alpha+1}-2 k|s|^{\beta+1}+(k-g) \dot{k} \\
= & -2 k|s|^{\alpha+1}-2 k|s|^{\beta+1}+2(k-g)|s|^{\alpha+1}+2(k-g)|s|^{\beta+1} \\
& -(k-g)^{\alpha+1}-(k-g)^{\beta+1} \\
= & -2 g|s|^{\alpha+1}-2 g|s|^{\beta+1}-(k-g)^{\alpha+1}-(k-g)^{\beta+1} .
\end{aligned}
$$

In the same way,

$$
\begin{aligned}
& \dot{V}<-m\left\{\left(\frac{1}{2} s^{2}\right)+\left[\frac{1}{2}(k-g)^{2}\right]\right\}^{(1 / 2)(\alpha+1)} \\
&-2^{((1-\beta) / 2)} n\left\{\left(\frac{1}{2} s^{2}\right)+\left[\frac{1}{2}(k-g)^{2}\right]\right\}^{(1 / 2)(\beta+1)}, \\
& m=\min \left\{2 \cdot 2^{(1 / 2)(\alpha+1)} g, 2^{(1 / 2)(\alpha+1)}\right\}, \\
& n=\min \left\{2 \cdot 2^{(1 / 2)(\beta+1)} g, 2^{(1 / 2)(\beta+1)}\right\}, \dot{V}<0 .
\end{aligned}
$$

\section{Numerical Simulation}

To verify the effect of the controller, numerical simulations were performed on MATLAB. In the simulations, the system parameters are shown in Table 1.

The control parameters are shown in Table 2 .

With the above parameters, the simulation results are as follows:

(1) Fixed-time sliding-mode control: we compare the response between fixed-time sliding-mode controller and finite-time sliding-mode controller (add the controller at $t=20 \mathrm{~s}$ ). The results are shown in Figure 1, where we can see that both the finite-time sliding-mode controller and the fixedtime sliding-mode controller can make the system stable in the limited time available, and the fixedtime controller can make the system stabilize faster. The control effect of the fixed-time slidingmode controller is much better than that of the finite-time controller.

(2) The response comparison between a non-faulttolerant controller and fault-tolerant controller: we compare the response between the non-faulttolerant controller and fault-tolerant controller (add the controller at $t=10 \mathrm{~s}$ ). The results are shown in Figure 2, where it can be seen that in the non-fault-tolerant control system, when the system executor fails, the system fluctuation is 


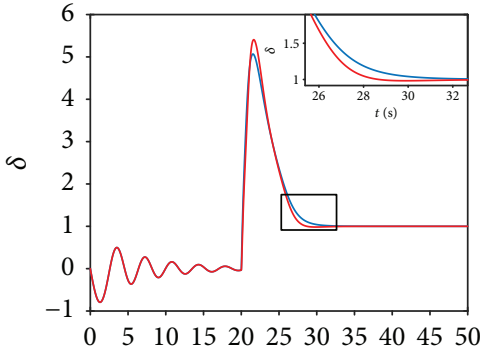

$t(\mathrm{~s})$

Finite time

(a) Power angle

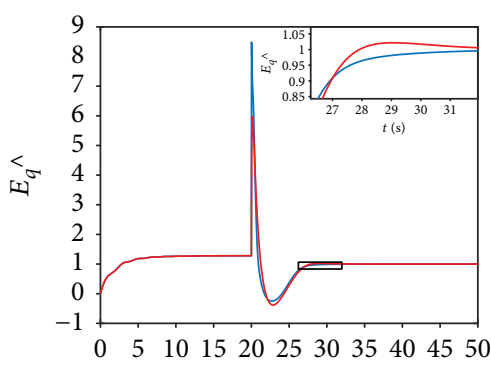

$t(\mathrm{~s})$

Finite time

Fixed time

(c) Transient emf

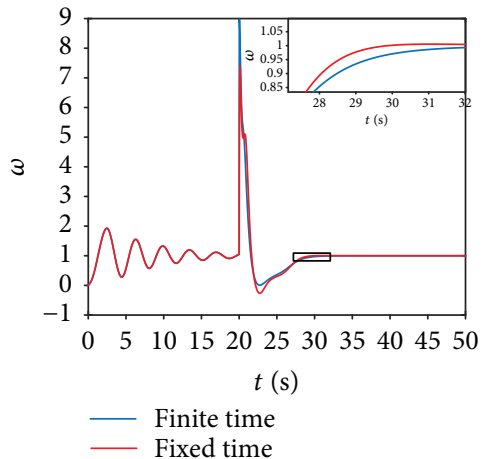

(b) Rotation speed

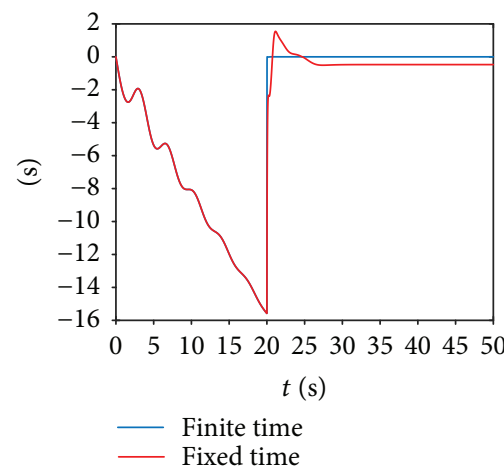

(d) Sliding surface

Figure 1: The comparison between fixed-time sliding-mode controller and finite-time sliding-mode controller.

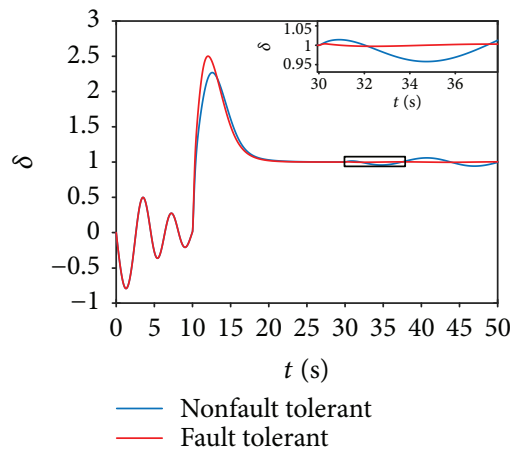

(a) Power angle

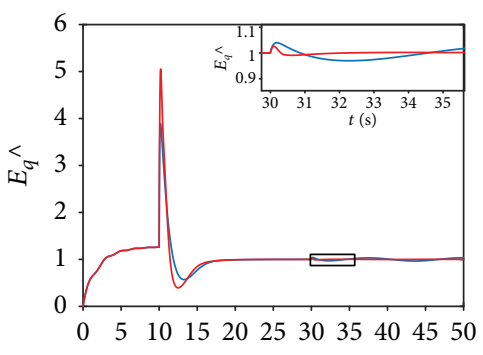

$t$ (s)

Nonfault tolerant

Fault tolerant

(c) Transient emf



Nonfault tolerant

Fault tolerant

(b) Rotation speed

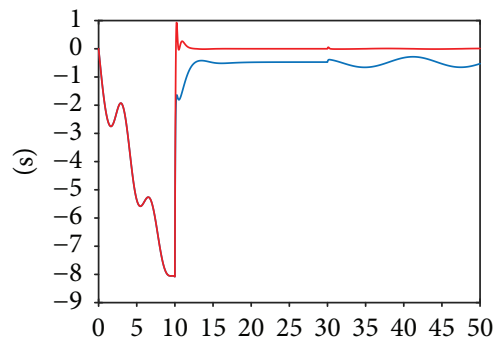

$t$ (s)

Nonfault tolerant

Fault tolerant

(d) Sliding surface

FIGURE 2: Simulation results. 


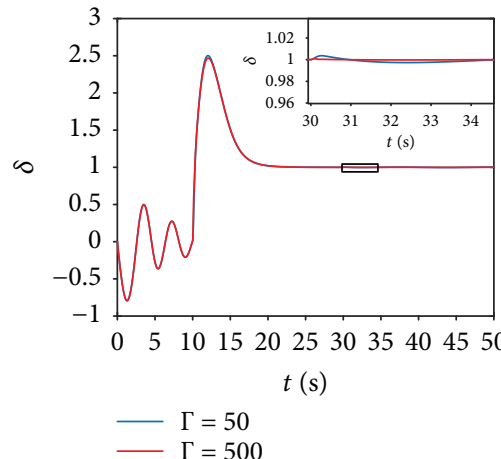

(a) Power angle

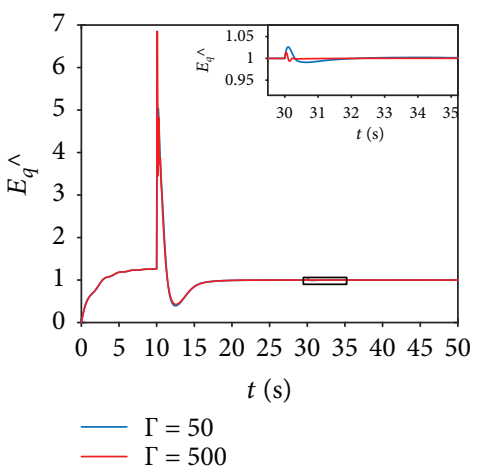

(c) Transient emf

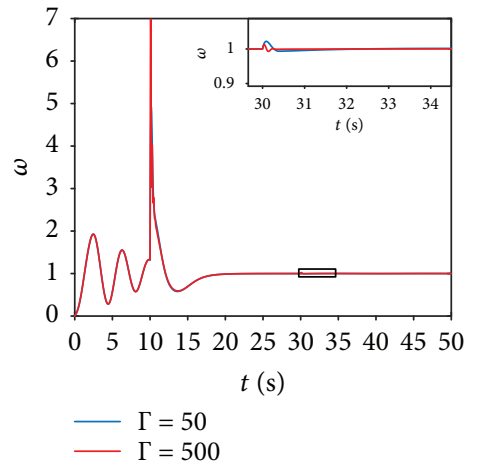

(b) Rotation speed

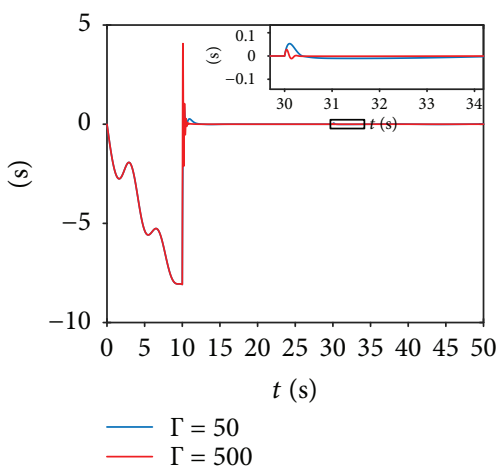

(d) Sliding surface

FIgURE 3: Simulation results.

more obvious, and the fault-tolerant system only has a very small fluctuation; furthermore, this fluctuation quickly disappears.

(3) Control effects of different parameters of $\Gamma$ : in order to optimize the control performance, the impact of the designed parameters on control performance is studied. To stabilize the system, we selected a big value for parameter $\Gamma$, and research has been done to verify it. The results are shown in Figure 3. It can be seen from Figure 3 that when the parameter $\Gamma$ becomes large, the control effect becomes better, which verifies the correctness of the theory.

\section{Conclusions}

In this paper, a coordinated excitation and control valve model of a waste heat generator with actuator failure was proposed. By designing a proportional integral sliding-mode surface, a sliding-mode control strategy was proposed to control the waste heat generator to a predetermined target. On this basis, using the fixed-time control theory, the corresponding law of fault estimation was designed for system-specific faults, and the sliding-mode fault-tolerant controller was constructed such that the system could still operate stably when actuator faults occurred and yielded an acceptable performance. The results of a numerical simulation showed that the proposed sliding-mode variable-structure control method is robust, has a short control transition time and a relatively smooth transition, and can greatly improve system stability, which well meets the requirements of control objectives. It can also be seen by magnifying the simulation image that the system has little buffeting and is completely within an acceptable range. But on the other hand, the study has some limitations. The control of this paper is only designed for the coordinated control of the excitation generator and the steam valve, which does not involve the dynamic influence of the boiler part of the waste heat power generation system. It may cause some deviations, which need further study on the multiobjective coordinated optimization control.

\section{Data Availability}

No data were used to support this study.

\section{Conflicts of Interest}

The authors declare that they have no conflicts of interest.

\section{Authors' Contributions}

Zhi Wang and Yateng Bai contributed equally to this work.

\section{References}

[1] G. Xu, Research on Intelligent Control Method of Synchronous Generator Excitation System, Chongqing Jiaotong University, 2014. 
[2] J. Jiang, T. Liu, and Y. Jing, "Nonlinear large-interference suppression controller for generator excitation system," Control Theory and Applications, vol. 10, pp. 1315-1322, 2013.

[3] H. Liu, H. Wei, and X. Li, "Nonlinear coordinated control with objective holographic feedbacks for large turbogenerator set," Transactions of China Electrotechnical Society, vol. 23, no. 4, pp. 25-31, 2008.

[4] Y. Chang, "Control of chaotic oscillation of power system based of feedback linearization method," Science Technology and Engineering, vol. 10, no. 25, pp. 6194-6199, 2010.

[5] R. Yang, R. Yuan, L. Wan, and H. Zhao, "Nonlinear robust voltage control for synchronous generators," Journal of Electrotechnics, vol. 27, no. 9, pp. 9-16,33, 2012.

[6] Z. Yu and X. Chen, "Nonlinear variable structure control theory and its application in power system," Automation of Electric Power Systems, vol. 21, no. 7, pp. 86-88, 1997.

[7] D. Xu, X. Li, and C. Hong, "Coordination control between TCSC and generator excitation based on optimal variable aim strategy," Power System Technology, vol. 32, no. 21, pp. 13-16,21, 2008.

[8] Y.-W. Liang, S.-D. Xu, and L.-W. Ting, "T-S model-based SMC reliable design for a class of nonlinear control systems," IEEE Transactions on Industrial Electronics, vol. 56, no. 9, pp. 3286-3295, 2009.

[9] Y. Wu, Sliding Mode Control Theory and Its Application in Mobile Manipulator, South China University of Technology, 2006.

[10] K. Zhang, Research on Robust Control Theory of Nonlinear Delay and Its Application in Turbo Generator, Harbin Institute of Technology, 2016.

[11] M. Blanke and M. Staroswiecki, Diagnosis and Fault-Tolerant Control, Springer-Verlag, Berlin, Germany, 2nd edition, 2006.

[12] R. Iserman, Fault-Diagnosis Systems. An Introduction from Fault Detection to Fault Tolerance, Springer-Verlag, Berlin, Germany, 2006.

[13] M. Rodrigues, D. Theilliol, and D. Sauter, "Active actuator fault tolerant control design for polytopic LPV systems," in presented at the International Federation Automatic Control (IFAC) Symposium Fault Detection, Supervision Safety Technical Processes, (SAFEPROCESS), Beijing, China, 2006.

[14] V. Lešić, M. Vašak, N. Perić, T. M. Wolbank, and G. Joksimović, "Fault-tolerant control of a wind turbine with a squirrel-cage induction generator and rotor bar defects," Automatika, vol. 54, no. 3, pp. 316-328, 2013.

[15] E. M. ElBeheiry, "Two tracking control problems applied to damping nonlinear vehicle vibrations," Journal of Sound and Vibration, vol. 321, no. 3-5, pp. 471-491, 2009.

[16] A. Polyakov, "Nonlinear feedback design for fixed-time stabilization of linear control systems," IEEE Transactions on Automatic Control, vol. 57, no. 8, pp. 2106-2110, 2012.

[17] S. P. Bhat and D. S. Bernstein, "Finite-time stability of continuous autonomous systems," SIAM Journal on Control and Optimization, vol. 38, no. 3, pp. 751-766, 2000.

[18] H. K. Khalil, Nonlinear Systems Third Edition, Prentice-Hall, Inc., Upper Saddle River, NJ, USA, 2002. 


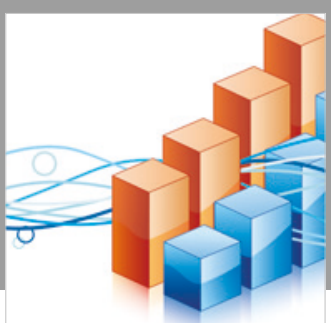

Advances in

Operations Research

\section{-n-m}
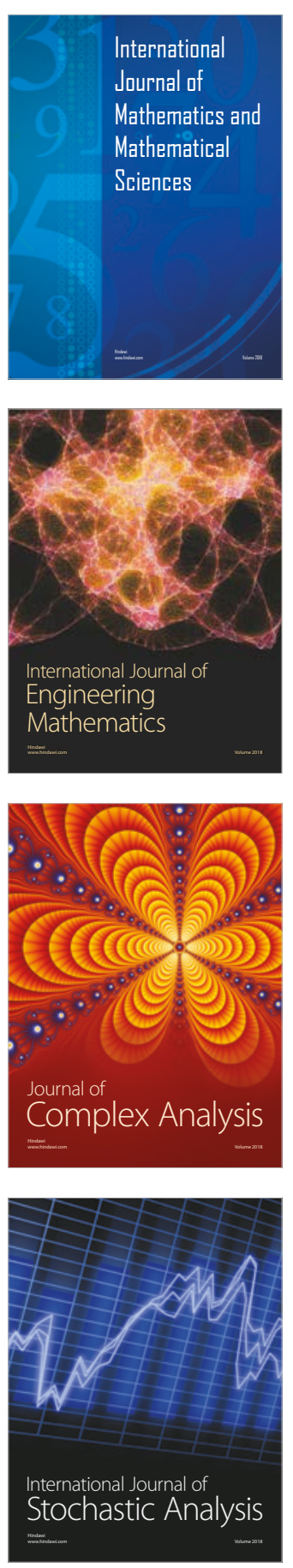
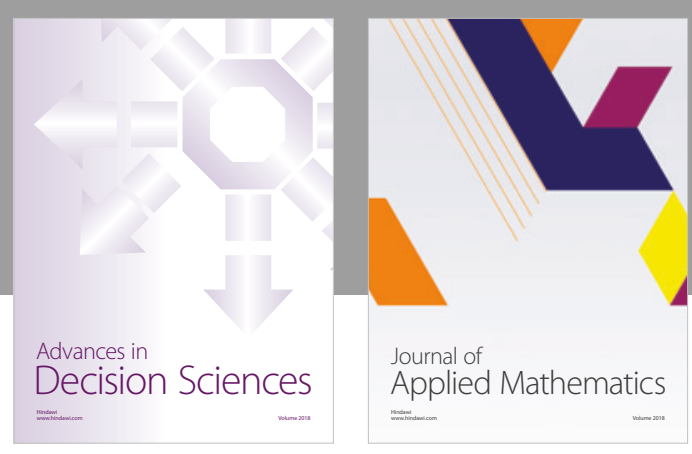

Journal of

Applied Mathematics


Submit your manuscripts at

www.hindawi.com

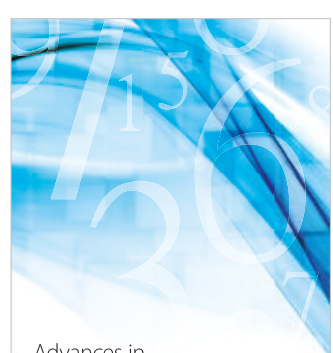

Advances in
Numerical Analysis
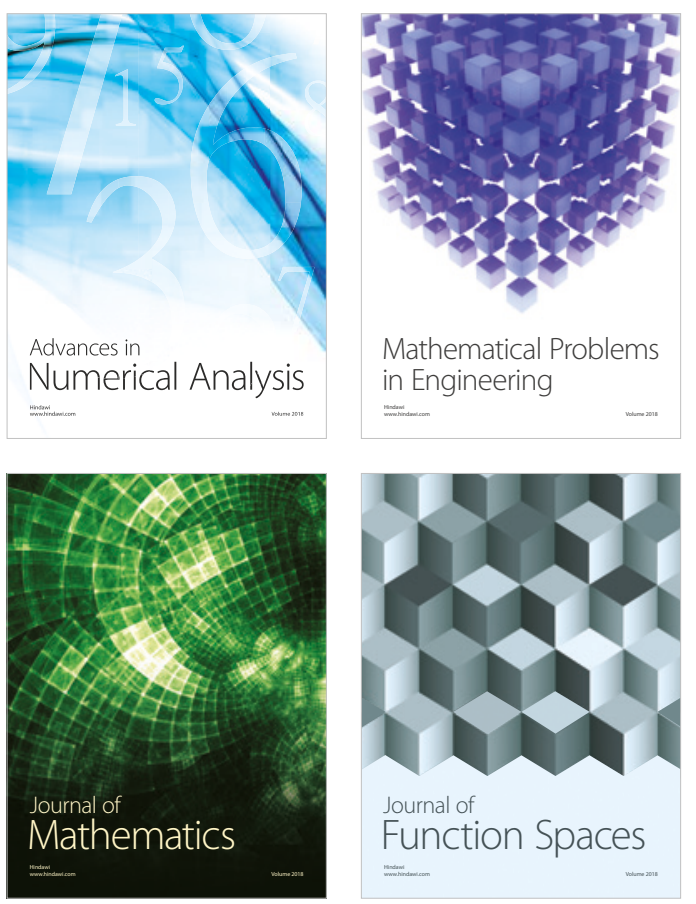

Mathematical Problems in Engineering

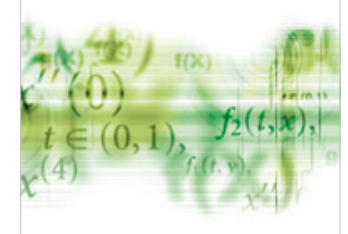

International Journal of

Differential Equations

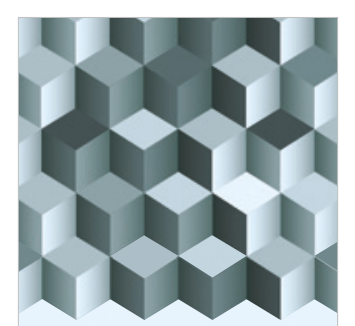

Journal of

Function Spaces
The Scientific

World Journal

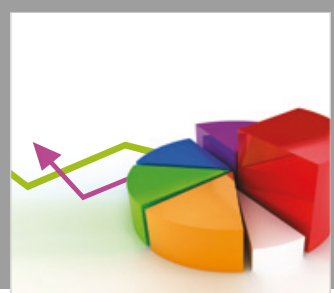

Journal of

Probability and Statistics
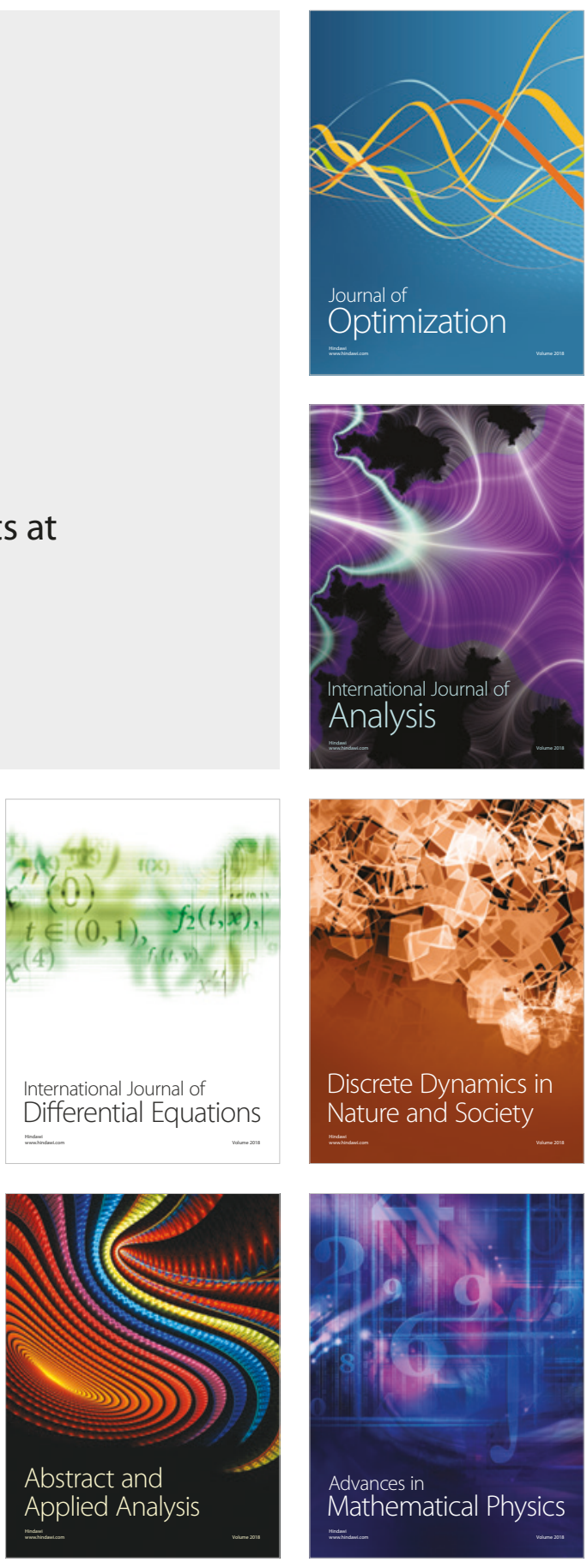